\title{
RADIOTHERAPY \\ A DOUBLE-EDGED SWORD FOR NSCLC?
}

New research has shown that disruption of tumours during radiotherapy in patients with non-small-cell lung cancer (NSCLC) can release viable cancer cells into the peripheral circulation-a possible mechanism underlying the formation of distant metastases.

Most deaths - even after radical treatment-associated with epithelial cancers are due to metastasis from the original tumour. "Radiotherapy can cause severe damage to the structure of a tumour. The rapid breakdown of a tumour could cause a flood of cancerous material, including intact cells, to enter the lymphatics," explains Michael MacManus, senior author of this study. He continues, "if our hypothesis is correct, it should be possible to detect circulating irradiated tumour cells."

To address this issue, the researchers, led by Olga Martin, took advantage of the well-known ability of the $\gamma \mathrm{H} 2 \mathrm{AX}$ antibody to detect radiation-induced double-stranded DNA breaks by binding to the phosphorylated histone $\mathrm{H} 2 \mathrm{AX}$. Given the sensitivity of the $\gamma \mathrm{H} 2 \mathrm{AX}$ assay, this was considered the ideal marker for the identification of circulating tumour cells (CTCs) derived from the irradiated mass. Indeed, the analysis of CTCs in the blood of patients with NSCLC revealed an increase of $\gamma \mathrm{H} 2 \mathrm{AX}$-positive tumour cells after fractionated radiotherapy. Further experiments showed that these CTCs were viable, as they proliferated in short-term culture and were able to form $\gamma \mathrm{H} 2 \mathrm{AX}$ foci in response to ex vivo irradiation (a sign that cells were trying to repair radiation-induced DNA damage).

These findings demonstrate that radiotherapy (especially in the early treatment stage, when the accumulated radiation dose is not sufficient to kill most cancer cells) can enable viable tumour cells to be released into the circulation, possibly leading to an increased risk of distant metastasis. "Although this possibility is of concern," says MacManus, "radiotherapy is indispensable for many cancers. Our findings offer hope that the risk of distant metastasis after curative irradiation might be reduced by novel therapeutic strategies, such as more-intense early radiation or co-administration of new or existing systemic agents."

Alessia Errico

Original article Martin, 0. A. et al. Mobilization of viable tumor cells into the circulation during radiation therapy. Int. J. Radiat. Oncol. Biol. Phys. doi:10.1016/ j.ijrobp.2013.10.033 